\title{
Phosphorylation of cMet tyrosine residues in murine ascitic hepatic cancer cell lines with different lymph node metastatic potentials
}

\author{
${\text { YING } \text { LI }^{2 *}, \text { XIAOHUA HUANG }}^{1,3^{*}}$, QIAOSHU ZHANG ${ }^{1}$ and KELI MA ${ }^{1}$ \\ ${ }^{1}$ Department of Biochemistry and Molecular Biology, Dalian Medical University, Liaoning 116044; \\ ${ }^{2}$ Department of Clinical Laboratory, Second Affiliated Hospital of Dalian Medical University, Liaoning 116023; \\ ${ }^{3}$ Department of Clinical Biochemistry, College of Laboratory Medicine, Dalian Medical University, \\ Dalian, Liaoning 116044, P.R. China
}

Received January 10, 2013; Accepted May 30, 2013

DOI: $10.3892 / \mathrm{mmr} .2013 .1527$

\begin{abstract}
The aim of the present study was to determine the molecular mechanism by which the hepatocyte growth factor (HGF) receptor (cMet) regulates lymphatic metastasis in hepatocellular carcinoma. Mouse hepatoma ascites cell lines with different lymph node metastatic potentials, Hca-F (high metastatic potential) and Hca-P (low metastatic potential), were cultured in vitro. Cells were treated with HGF, fibronectin (FN) and laminin (LN), and the phosphorylated tyrosine residues of cMet and the activities of intracellular phospholipase $\mathrm{C} \gamma /$ diacylglycerol/protein kinase C (PLC $\gamma / \mathrm{DAG} / \mathrm{PKC})$ and phosphoinositol-3-kinase/protein kinase B (PI3K/AKT) signaling pathways were analyzed comparatively in the two cell lines using western blot analysis and migration assays. Following HGF treatment, the phosphorylation of cMet at Tyr 1313 and 1365 in Hca-F cells was higher, while the phosphorylation of cMet at Tyr 1349 was lower than that in Hca-P. The activity of PLC $\gamma / \mathrm{DAG} / \mathrm{PKC}$ was increased in Hca-F cells compared with Hca-P cells, whereas the activity of PI3K/AKT was reduced. After FN treatment, the phosphorylation of cMet at Tyr 1313 and the activity of the PLC $\gamma / \mathrm{DAG} / \mathrm{PKC}$ signaling pathway was increased in Hca-F cells compared with Hca-P cells. Following LN treatment, the phosphorylation of cMet at Tyr 1365 and the activity of PLC $\gamma / \mathrm{DAG} / \mathrm{PKC}$ was higher in $\mathrm{Hca}-\mathrm{F}$ cells than in Hca-P cells. Results of the current study
\end{abstract}

Correspondence to: Professor Keli Ma, Department of Biochemistry and Molecular Biology, 9 Lushun Southwest Road, Dalian Medical University, Dalian, Liaoning 116044, P.R. China

E-mail: makelipaper@163.com

*Contributed equally

Abbreviations: PI3K, phosphoinositol-3-kinase; AKT, protein kinase B; PLC $\gamma 1$, phospholipase $\gamma 1$; DAG, diacylglycerol; $\mathrm{PKC}$, protein kinase $\mathrm{C}$

Key words: liver cancer, metastasis, hepatocyte growth factor receptor, PLC $\gamma$, AKT indicate that a number of ligands stimulate the phosphorylation of cMet at various tyrosine residues, activating different signaling transduction pathways. In addition, the same ligand was observed to phosphorylate different tyrosine residues on cMet in the two cell lines, as well as activate different intracellular signaling transduction pathways. After cMet is activated, various tyrosine residues are phosphorylated, leading to the activation of the PI3K/AKT and PLC $\gamma / \mathrm{DAG} / \mathrm{PKC}$ signaling pathways to different extents in the two cells lines. These results may be important in determining the lymph node metastatic potentials of the two cell lines.

\section{Introduction}

The prevention and reduction of invasion and metastasis in malignant tumors is extremely important and these processes represent the main cause of clinical mortality. An important method of metastasis occurs via the lymphatic system. Tumor metastasis is a complex process that involves genetic abnormalities in tumor cells, changes in the signal regulation system, invasiveness, adhesion and angiogenesis (1). To improve understanding of the effect of regulating the function and expression of metastasis-associated molecules on the molecular mechanism of tumor metastasis, drug targets and anti-metastatic drugs must be identified and screened for in order to inhibit tumor metastasis and reduce the mortality rate of cancer patients.

Hepatocyte growth factor (HGF), also known as mesenchymal factor, is a cytokine produced in stromal cells, including fibroblasts and macrophages. HGF targets adjacent cells in a paracrine manner, binding to the cell surface receptor and activating tyrosine kinases, and is important for the regulation of cell growth and migration (2).

The HGF receptor is also known as cMet, as it is the product of the proto-oncogene, $c$ Met. cMet possesses tyrosine kinase activity and is a transmembrane receptor protein. HGF recognizes and binds to cMet, which leads to receptor dimerization and the phosphorylation of tyrosine residues in intracellular domains. Intracellular signaling molecules containing SH2 domains recognize the phosphorylation of 
these tyrosine residues, activating various intracellular signal transduction pathways. There are at least three known signal transduction pathways activated by cMet, including phospholipase $C \gamma /$ diacylglycerol/protein kinase C (PLC $\gamma / D A G / P K C)$, phosphoinositol-3-kinase/phosphoinositol-dependent protein kinase/protein kinase B (PI3K/PDK/PKB or PI3K/AKT) and mitogen-activated protein kinase (MAPK). Abnormal PI3K/AKT and PLC $\gamma / D A G / P K C$ signaling pathways have been previously reported to be markedly associated with tumorigenesis, particularly invasion and metastasis (3).

cMet is activated by molecules in the extracellular matrix, including laminin (LN) and the cell surface adhesion molecule fibronectin (FN) (4-7). During invasion and metastasis, LN may activate cMet and promote the expression and activation of proteolytic enzymes, including collagenase IV, matrix metalloproteinases and urokinase-type plasminogen activator. FN, on the surface of the cells of the target organ tissue, may also activate cMet, thereby promoting the expression of vascular endothelial growth factor, which may specifically promote endothelial cell division and increase vascular permeability, followed by the stimulation of tumor angiogenesis, alteration of the tumor stroma and promotion of the growth of secondary tumors (8). In the present study, the molecular mechanisms of cMet-mediated cell signal transduction in the regulation of tumor metastasis were analyzed in two mouse ascites hepatoma cell lines associated with different lymphatic metastatic potentials, Hca-F (high metastatic potential) and Hca-P (low metastatic potential). The phosphorylation of tyrosine residues of cMet and the activation of the intracellular PI3K/AKT and PLC $\gamma /$ DAG/PKC signaling pathways following stimulation with different ligands were analyzed and compared between the two cell lines.

\section{Materials and methods}

Materials. Primary antibodies anti-p-Met (Tyr 1313) IgG, anti-p-Met (Tyr 1349) IgG, anti-p-Met (Tyr 1365) IgG, anti-p-PLC $\gamma 1$ (Tyr 783) IgG, anti-PLC $\gamma 1$ IgG, anti-p-Akt1/2/3 (Thr 308) IgG, anti-p-Akt1/2/3 (Ser 473) IgG and anti- $\beta$-actin IgG, biotinylated anti-mouse, -rabbit and -goat secondary antibodies and alkaline phosphatase-labeled streptavidin were purchased from Santa Cruz Biotechnology, Inc. (Santa Cruz, CA, USA). BCIP/NBT chromogenic agents were obtained from Wuhan Boster Biological Technology, Ltd. (Wuhan, China)

Reagents. Recombinant human HGF was obtained from Peprotech (Rocky Hill, NJ, USA). LN and FN were obtained from Sigma-Aldrich (St. Louis, MO, USA). Inhibitors were obtained from Calbiochem (San Diego, CA, USA; U73122) or Sigma-Aldrich (LY294002).

Cell culture. For all experiments, Hca-P and Hca-F cell lines were grown in multi-well plates in RPMI-1640 medium supplemented with $10 \%$ heat-inactivated FBS, $100 \mathrm{U} / \mathrm{ml}$ penicillin and $100 \mathrm{mg} / \mathrm{ml}$ streptomycin under a $5 \% \mathrm{CO}_{2}$ atmosphere at $37^{\circ} \mathrm{C}$.

Analysis of tyrosine phosphorylation of cMet in the cell lines. Culture medium was removed and the cells were washed twice in serum-free RPMI-1640 medium and incubated overnight in serum-free medium. Cells were collected, divided into the control and treatment groups and added to serum-free medium containing $20 \mathrm{ng} / \mathrm{ml} \mathrm{HGF,} 2 \mathrm{ng} / \mathrm{ml} \mathrm{FN}$ or $2 \mathrm{ng} / \mathrm{ml}$ $\mathrm{LN}$ for $10 \mathrm{~min}$ at room temperature. Centrifugal supernatants were discarded and the cells were lysed in $200 \mu 1$ RIPA buffer (1\% Triton $\mathrm{X}-100,150 \mathrm{mM} \mathrm{NaCl}, 25 \mathrm{mM}$ Tris at $\mathrm{pH} \mathrm{7.5,}$ $0.5 \%$ sodium deoxycholate, $0.1 \% \mathrm{SDS}, 5 \mathrm{mM}$ pyrophosphate and $50 \mathrm{mM} \mathrm{NaF}$ ) containing $1 \mathrm{mM} \mathrm{Na}_{3} \mathrm{VO}_{4}, 1 \mathrm{mM}$ DTT, $1 \%$ protease inhibitor cocktail and $1 \%$ phosphatase inhibitor cocktail. Lysates were electrophoresed on a $10 \%$ SDS-PAGE gel. Next, proteins were transferred to PVDF membranes at $100 \mathrm{~mA}$ for $2 \mathrm{~h}$. Non-specific binding on the PVDF membranes was blocked with $3 \%$ (w/v) BSA. Target protein bands on the PVDF membranes were revealed by immunoblotting with anti-p-Met (Tyr 1313) IgG, anti-p-Met (Tyr 1349) IgG or anti-p-Met (Tyr 1365) IgG primary antibodies, biotinylated goat anti-rabbit IgG, horse anti-mouse IgG or rabbit anti-goat IgG secondary antibodies, alkaline phosphatase-labeled streptavidin and BCIP/NBT chromogenic agent.

Analysis of signal transduction pathway activity in the cell lines. Total protein from treated cells was analyzed by western blot analysis as described. Target protein bands in PVDF membranes were revealed by immunoblotting with anti-P-Akt1/2/3 (Thr 308) IgG, anti-P-Akt 1/2/3 (Ser 473) IgG, anti-PLC $\gamma 1$ IgG, anti-p-PLC $\gamma 1$ (Tyr 783) IgG, biotinylated goat anti-rabbit IgG, horse anti-mouse IgG or rabbit anti-goat IgG secondary antibodies, alkaline phosphatase-labeled streptavidin and BCIP/NBT chromogenic agents.

In vitro migration assay. Migration assays were performed using a Boyden chamber (Costar, Cambridge, MA, USA) with $8-\mu \mathrm{m}$ pore polycarbonate filters (BD Biosciences, Franklin Lakes, NJ, USA). Following overnight incubation in serum-free medium, cells $\left(1 \times 10^{5}\right)$ were resuspended in $300 \mu \mathrm{l}$ medium with $20 \mathrm{ng} / \mathrm{ml} \mathrm{HGF,} 2 \mathrm{ng} / \mathrm{ml} \mathrm{FN}$ or $2 \mathrm{ng} / \mathrm{ml} \mathrm{LN}$ and placed in the top chamber and $250 \mu 110 \%$ FBS medium was placed in the bottom chamber. After incubation for $6 \mathrm{~h}$ at $37^{\circ} \mathrm{C}$ in $5 \% \mathrm{CO}_{2}$, cells on the top membrane surface were mechanically removed. Cells that migrated to the bottom side of the membrane were fixed and stained with $0.1 \%$ DAPI (Sigma-Aldrich). Images were captured and stained cells were counted under a microscope in five randomly selected fields. PLC $\gamma / \mathrm{DAG} / \mathrm{PKC}$ and PI3K/AKT pathways were blocked with $10.5 \mu \mathrm{M}$ U73122 and $15 \mu \mathrm{M}$ LY294002 for $4 \mathrm{~h}$, respectively. Cells were then harvested and used for migration assays.

Statistical analysis. All results were repeated in at least three independent experiments and consistently yielded similar results. Data were analyzed by the Gel-Pro analyzer (Media Cybernetics, USA) and GraphPad Prism 5 (Beijing, China). $\mathrm{P}<0.05$ was considered to indicate a statistically significant difference. Results are presented as the mean \pm SEM.

\section{Results}

Effect of HGF on tyrosine phosphorylation of cMet and signaling pathway activities. cMet is activated through the ligand binding to the receptor, receptor dimerization and 
A
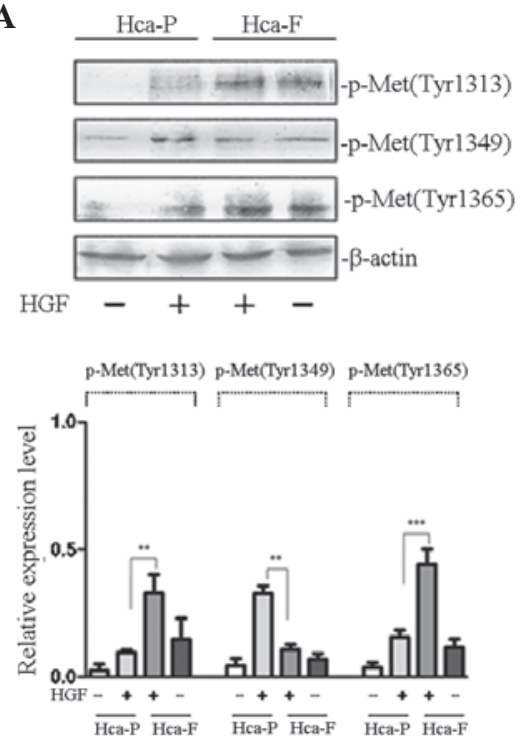

B
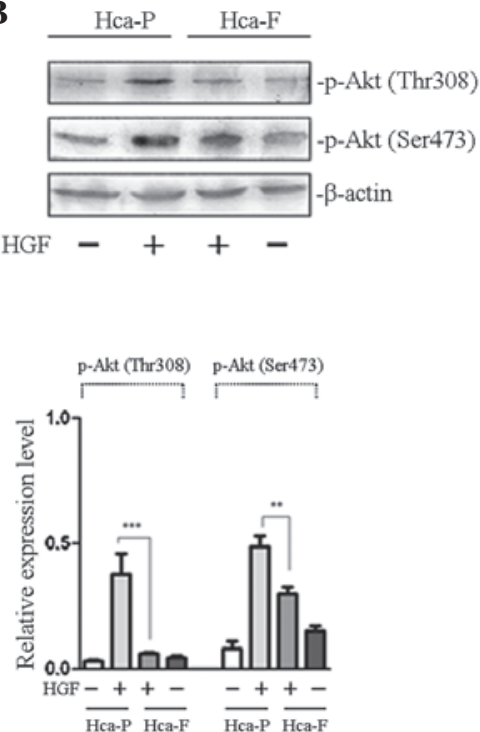
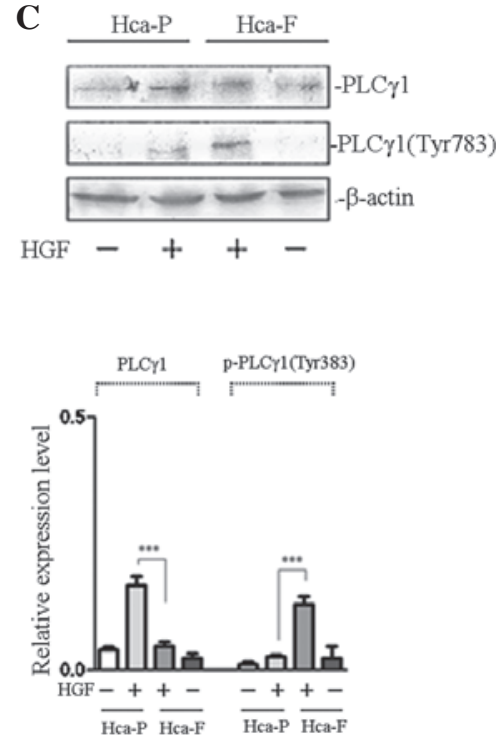

Figure 1. Effect of HGF on the phosphorylation of cMet and signaling pathway activity in Hca-P and Hca-F cells. (A) Effect of HGF on the phosphorylation of cMet. (B) Effect of HGF on the activity of PI3K/AKT signaling pathway. (C) Effect of HGF on the activity of PLC $\gamma /$ DAG/PKC signaling pathway. Cells were stimulated with HGF and subjected to western blot analysis with anti-p-Met (Tyr 1313), anti-p-Met (Tyr 1365), anti-p-Akt 1/2/3 (Ser 473), anti-p-Akt1/2/3 (Thr 308), anti-PLC $\gamma 1$ and anti-p-PLC $\gamma 1$ (Tyr 783) antibodies. The phosphorylation of cMet at Tyr 1313 and 1365 in Hca-F cells was higher than that in Hca-P cells, while phosphorlyation of Tyr 1349 was lower than that in Hca-P cells. The activity of the PLC $\gamma 1 / \mathrm{DAG} / \mathrm{PKC}$ signaling pathway was greater in Hca-F cells than that in Hca-P cells; however, the activity of PI3K/AKT was lower. HGF, hepatocyte growth factor; cMet, HGF receptor; Hca-F, highly metastatic cell line; Hca-P, low metastatic cell line; PI3K, phosphoinositol-3-kinase; Akt, protein kinase B; PLC $\gamma 1$, phospholipase $\gamma 1$; DAG, diacylglycerol; PKC, protein kinase C.

phosphorylation of intracellular tyrosine residues. Different phosphorylated tyrosine residues activate different downstream intracellular signaling pathways, which then play various roles in the regulation of a number of biological functions, including proliferation, invasion and metastasis (9). As demonstrated in Fig. 1A, HGF-stimulated phosphorylation of cMet at Tyr 1313 and 1365 in highly metastatic Hca-F cells was higher than that in low metastatic Hca-P cells; however, phosphorylation at Tyr 1349 was lower than that in the Hca-P cells.

Growth factor receptors activate PI3K/AKT signal transduction, an important pathway involved in the regulation of tumor metastasis. AKT functions as a protein kinase in PI3K/AKT signal transduction and the phosphorylation of AKT at Thr 308 and Ser 473 is activated by its upstream kinase, PDK (10). Therefore, the detection of intracellular phosphorylated AKT levels may be used to determine the activity of PI3K/AKT signal transduction. To study the effect of AKT phosphorylation in the two cell lines treated with HGF, the phosphorylation of AKT at Thr 308 and Ser 473 was observed. As revealed in Fig. 1B, HGF-stimulated phosphorylation of AKT at Thr 308 and Ser 473 in low metastatic Hca-P cells was higher than that in highly metastatic Hca-F cells. These results indicated that PI3K/AKT signaling pathway activity was greater in low metastatic Hca-P than in highly metastatic Hca-F cells.

PLC $\gamma /$ DAG/PKC signal transduction is another important pathway involved in the regulation of tumor metastasis. When the growth factor receptor is activated, the $\mathrm{SH} 2$ domain of PLC $\gamma 1$ identifies a specific phosphorylated tyrosine residue and binds to the receptor. Receptor tyrosine kinase catalyzes the phosphorylation of PLC $\gamma 1$ at Tyr 783, thereby activating PLC $\gamma / D A G / P K C$ signal transduction (11). To study the effect of PLC $\gamma 1$ phosphorylation in the two cell lines treated with
HGF, the phosphorylation of PLC $\gamma 1$ at Tyr 783 was observed. As demonstrated in Fig. 1C, HGF-stimulated phosphorylation of PLC $\gamma 1$ at Tyr 783 in highly metastatic Hca-F cells was higher than that in low metastatic Hca-P cells. These results indicated that PLC $\gamma / \mathrm{DAG} / \mathrm{PKC}$ signaling pathway activity was greater in Hca-F than in Hca-P cells.

Effect of FN on the tyrosine phosphorylation of cMet and signaling pathway activities. To investigate the effect of FN on the phosphorylation of cMet, phosphorylation of cMet at Tyr 1313, 1349 and 1365 in Hca-F and Hca-P cells was analyzed. Fig. 2A reveals that, following FN treatment, the phosphorylation of cMet at Tyr 1313 in highly metastatic Hca-F cells was significantly higher compared with low metastatic Hca-P cells; however, the phosphorylation of cMet at Tyr 1349 and 1365 was not found to be significantly different between the two cell lines.

To study the effect of FN on the activity of the PI3K/AKT and PLC $\gamma / \mathrm{DAG} / \mathrm{PKC}$ signaling pathways of the two cell lines, the phosphorylation of Akt at Thr 308 and Ser 473 and PLC $\gamma$ at Tyr 783 was observed. No significant differences in the phosphorylation of Akt at Thr 308 and Ser 473 were found between Hca-P and Hca-F cells (Fig. 2B). The phosphorylation of PLC $\gamma 1$ at Tyr 783 in highly metastatic Hca-F cells was higher than that in low metastatic Hca-P cells (Fig. 2C). These results indicated that there was no significant difference in PI3K/AKT signaling pathway activity between the two cell lines, whereas PLC $\gamma / \mathrm{DAG} / \mathrm{PKC}$ activity was greater in Hca-F cells than that in Hca-P cells.

Effect of LN on the tyrosine phosphorylation of cMet and on signaling pathway activities. To investigate the effect of FN on the phosphorylation of cMet, phosphorylation at Tyr 1313, 1349 
A
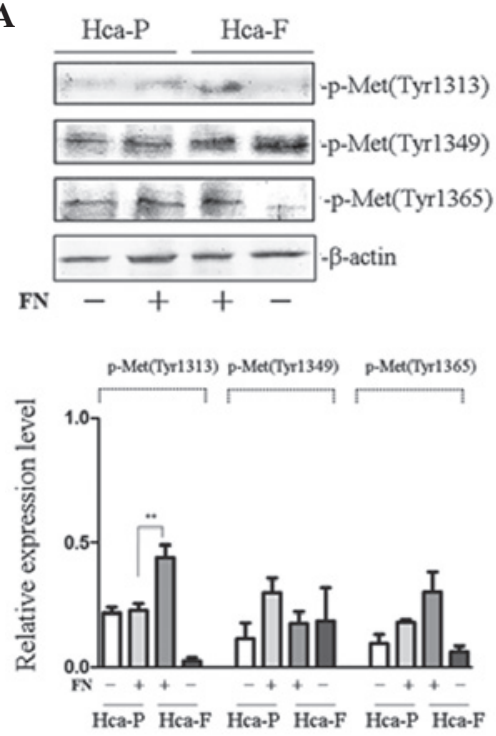

B
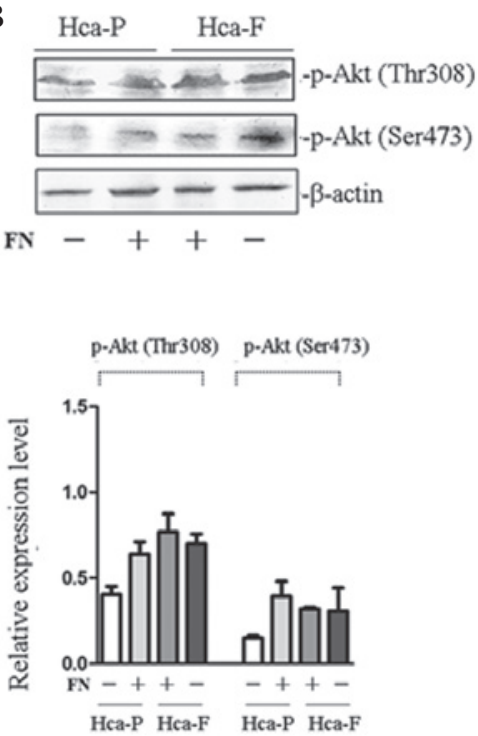
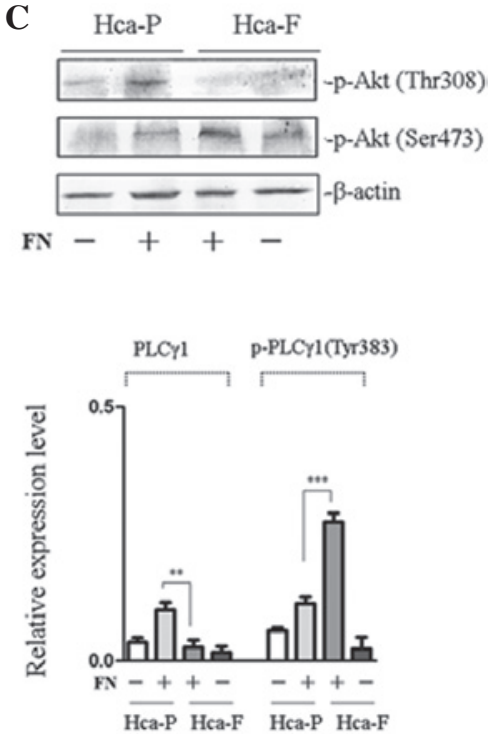

Figure 2. Effect of FN on the phosphorylation of cMet and signaling pathway activity in Hca-P and Hca-F cells. (A) Effect of FN on the phosphorylation of cMet. (B) Effect of FN on the activity of PI3K/AKT signaling pathway. (C) Effect of FN on the activity of PLC $\gamma / \mathrm{DAG} / \mathrm{PKC}$ signaling pathway. Cells were stimulated with FN and subjected to western blot analysis with anti-p-Met (Tyr 1313), anti-p-Met (Tyr 1365), anti-p-Akt 1/2/3 (Ser 473), anti-p-Akt1/2/3 (Thr 308), anti-PLC $\gamma 1$ (1249) and anti-p-PLC $\gamma 1$ (Tyr 783) antibodies. The phosphorylation of cMet at Tyr-1313 in Hca-F cells was higher than that in Hca-P cells and the activity of the PLC $\gamma 1 / \mathrm{DAG} / \mathrm{PKC}$ signaling pathway was greater in Hca-F cells than that in Hca-P cells. FN, fibronectin; cMet, HGF receptor; Hca-F, highly metastatic cell line; Hca-P, low metastatic cell line; Akt, protein kinase B; PLC $\gamma 1$, phospholipase $\gamma 1$; DAG, diacylglycerol; PKC, protein kinase C.

A
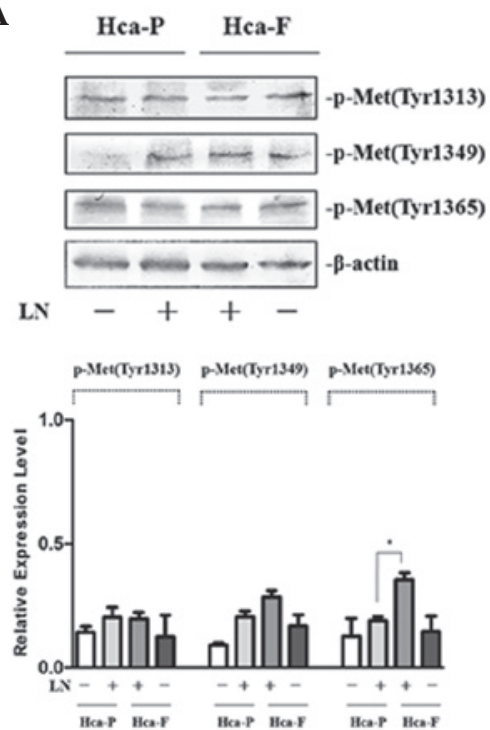

B
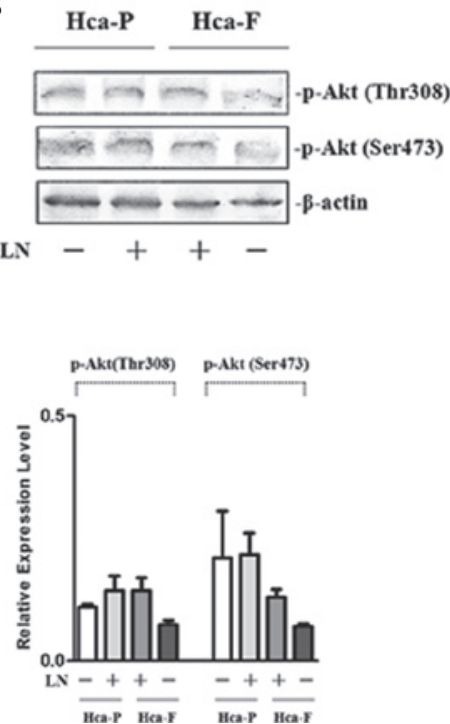
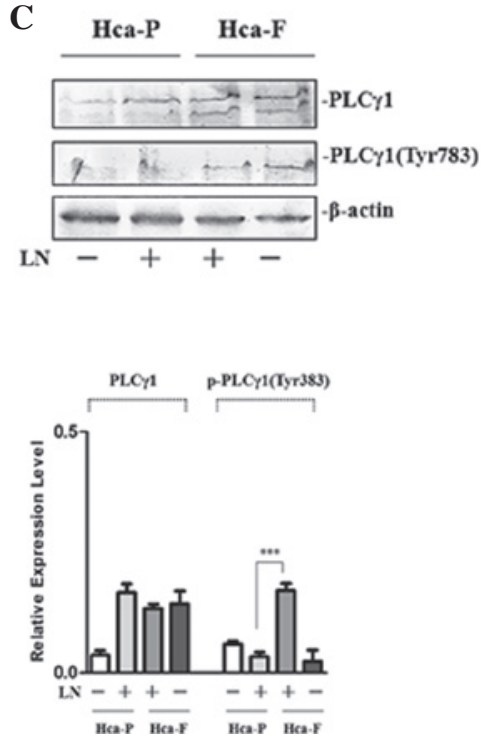

Figure 3. Effect of LN on the phosphorylation of cMet and signaling pathway activity in Hca-P and Hca-F cells. (A) Effect of LN on the phosphorylation of cMet. (B) Effect of LN on the activity of PI3K/AKT signaling pathway. (C) Effect of LN on the activity of PLC $\gamma / \mathrm{DAG} / \mathrm{PKC}$ signaling pathway. Cells were stimulated with LN and subjected to western blot analysis with anti-p-Met (Tyr 1313), anti-p-Met (Tyr 1365), anti-p-Akt 1/2/3 (Ser 473), anti-p-Akt1/2/3 (Thr 308), anti-PLC $\gamma 1$ (1249) and anti-p-PLC $\gamma 1$ (Tyr 783) antibodies. The phosphorylation of cMet at Tyr 1365 in Hca-F cells was higher than that in Hca-P cells and the activity of PLC $\gamma 1 / \mathrm{DAG} / \mathrm{PKC}$ signaling in Hca-F cells was greater than that in Hca-P cells. LN, laminin; cMet, HGF receptor; Hca-F, highly metastatic cell line; Hca-P, low metastatic cell line; Akt, protein kinase B; PLC $\gamma 1$, phospholipase $\gamma 1$; DAG, diacylglycerol; PKC, protein kinase C.

and 1365 was analyzed in Hca-F and Hca-P cells. Following LN treatment, the phosphorylation of cMet at Tyr 1365 in highly metastatic Hca-F cells was observed to be significantly greater than that in low metastatic Hca-P cells. In addition, analysis of the phosphorylation of cMet at Tyr 1313 and 1349 revealed no significant differences between the two cell lines (Fig. 3A).

To study the effect of LN on the PI3K/AKT and PLC $\gamma /$ DAG/PKC activity of the two cell lines, phosphorylation of Akt at Thr 308 and Ser 473 and PLC $\gamma$ at Tyr 783 was observed. Following LN treatment, the phosphorylation of Akt at Thr 308 and Ser 473 was not identified to be significantly different between Hca-P and Hca-F cells (Fig. 3B); however, the phosphorylation of PLC $\gamma 1$ at Tyr 783 in highly metastatic Hca-F cells was higher than that in low metastatic Hca-P cells (Fig. 3C). These results indicated that PI3K/AKT activity is not significantly different between the two cell lines and that PLC $\gamma /$ DAG/PKC activity was greater in Hca-F cells compared with Hca-P cells. 
A

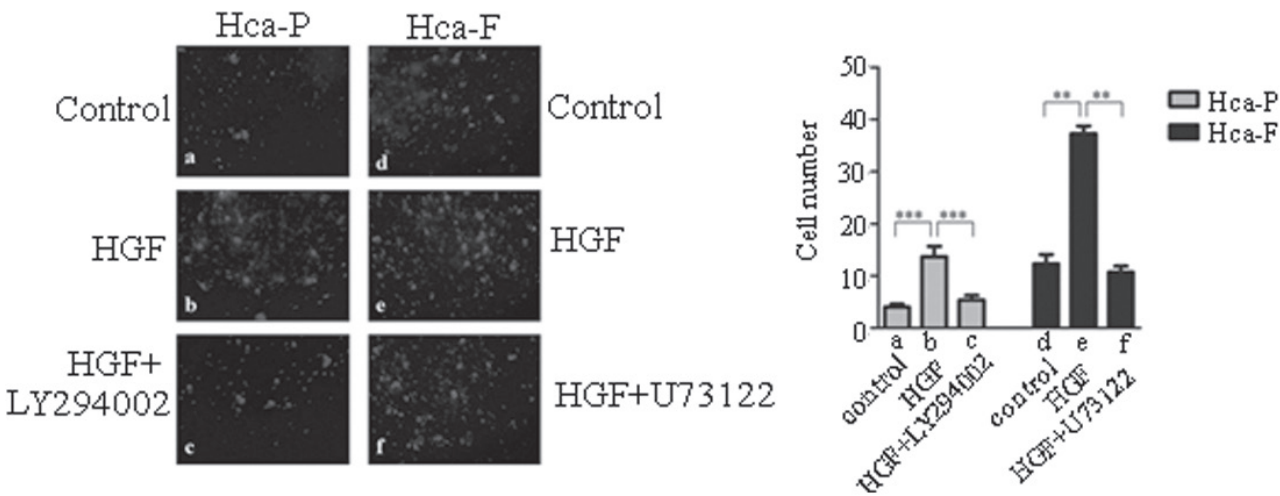

B
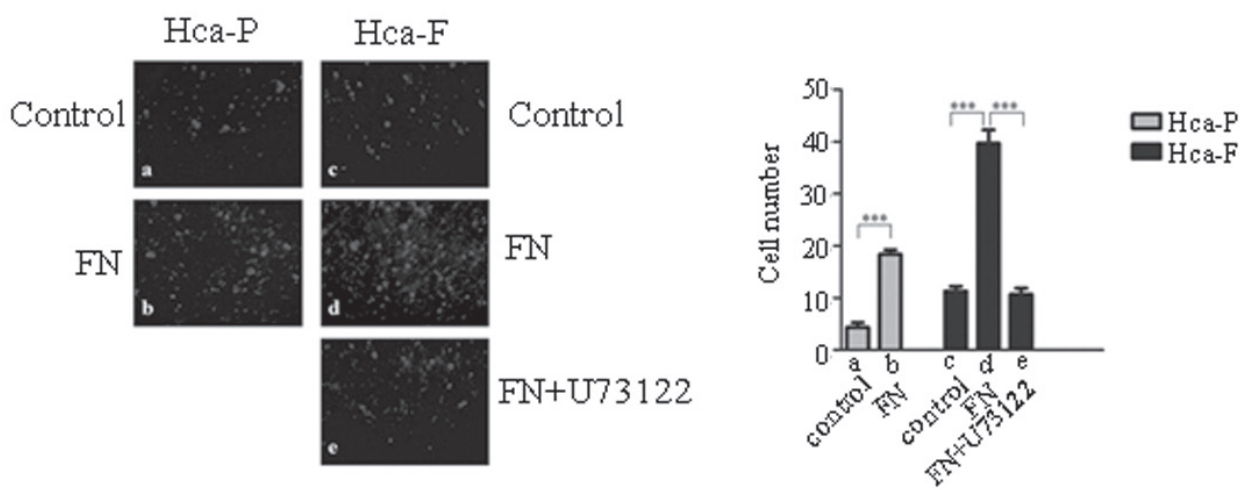

C
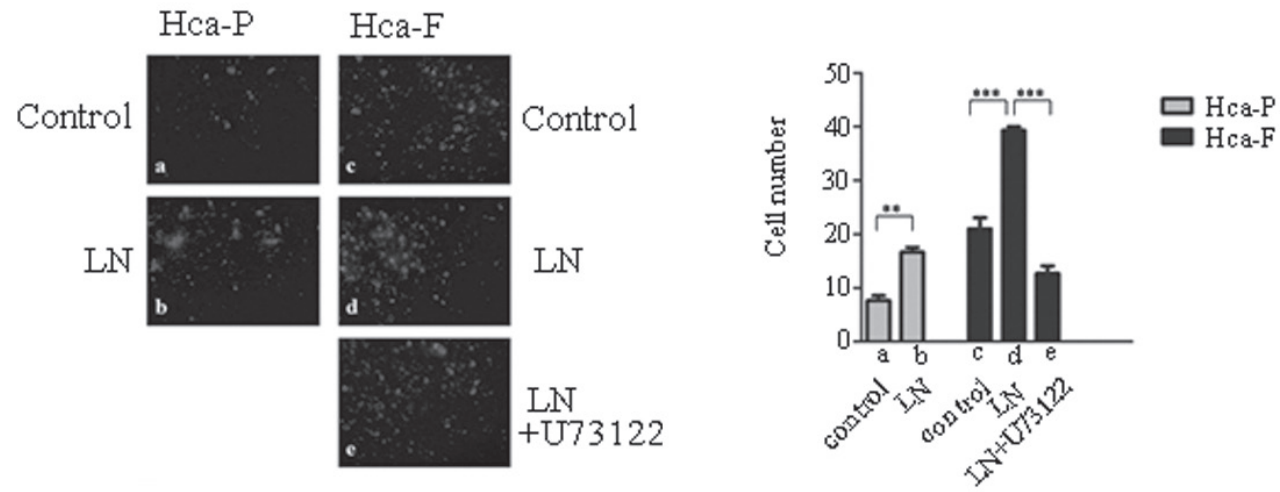

Figure 4. Effect of HGF, FN and LN on the migration of Hca-P and Hca-F cells. (A) Effect of HGF on the migration of Hca-P and Hca-F cells. (B) Effect of FN on the migration of Hca-P and Hca-F cells. (C) Effect of LN on on the migration of Hca-P and Hca-F cells. Cells were treated with HGF, FN or LN, PI3K/Akt and PLC $\gamma 1 / D A G / P K C$ signaling was blocked with LY294002 or U73122, respectively, and cells were then subjected to a migration assay. Membranes were fixed, stained and counted using fluorescence microscopy (magnification, x100). HGF, hepatocyte growth factor; FN, fibronectin; LN, laminin; Hca-F, highly metastatic cell line; Hca-P, low metastatic cell line; PI3K, phosphoinositol-3-kinase; Akt, protein kinase B; PLC $\gamma 1$, phospholipase $\gamma 1$; DAG, diacylglycerol; $\mathrm{PKC}$, protein kinase $\mathrm{C}$.

Effect of $H G F, F N$ and $L N$ on cell motility and migration in vitro. $\mathrm{PI} 3 \mathrm{~K} / \mathrm{AKT}$ and $\mathrm{PLC} \gamma / \mathrm{DAG} / \mathrm{PKC}$ signaling are major pathways by which cMet modulates cell motility and migration (12). The effect of HGF, FN and LN on cell motility and migration in vitro were investigated. As demonstrated in Fig. 4, HGF, FN and LN promoted Hca-P and Hca-F cell motility and migration.

Effect of blocking PI3K/AKT and PLC $\gamma / D A G / P K C$ signaling pathways on cell motility and migration. To determine whether cell motility and migration are mainly regulated by the PI3K/AKT or PLC $\gamma / \mathrm{DAG} / \mathrm{PKC}$ signaling pathways, the activity of PI3K in the cells was blocked with LY294002, an inhibitor of PI3K, and the activity of the PLC $\gamma / \mathrm{DAG} / \mathrm{PKC}$ signaling pathway in the cells was blocked with U73122. Blocking PI3K/AKT signaling with LY294002 reversed the positive effect of HGF on HGF-stimulated motility and migration in Hca-P cells in vitro (Fig. 4Aa-c). In Hca-F cells, blocking the PLC $\gamma / \mathrm{DAG} / \mathrm{PKC}$ signaling pathway with $\mathrm{U} 73122$ reversed the positive effects of HGF (Fig. 4Ad-f), FN (Fig. 4Bc-e) or LN (Fig. 4Cc-e) on HGF-, FN- or LN-stimulated motility and migration in vitro, respectively. These results indicated that HGF, FN and LN modulated cell motility and migration via the PLC $\gamma /$ DAG/PKC signaling pathway in Hca-F cells, and that HGF modulated cell motility and migration via the PI3K/AKT signaling pathway in Hca-P cells.

\section{Discussion}

HGF has a number of important biological functions, including the promotion of cell proliferation, inhibition of cell adhesion and increasing the expression of urokinase-type plasminogen 
activator receptor, which promotes the degradation of the extracellular matrix. In addition, HGF increases cytoskeletal protein phosphorylation and the destruction of the cytoskeleton, which makes it easier for cells to be deformed and migrate. Therefore, HGF is also known to promote mitogen, promoting separation factor, cell morphogen and migration factor in cell motility and migration. Not only is HGF important for the regulation of embryonic development, organ formation and cell growth, the growth factor also has a marked association with tumorigenesis and tumor progression, invasion, proliferation and metastasis (9).

HGF mediates these effects via its cognate receptor, cMet . cMet is a single transmembrane receptor protein that possesses tyrosine kinase activity. Upon ligand binding, cMet undergoes dimerization and phosphorylates key tyrosine residues. The intracellular SH2 domains of signaling molecules recognize the phosphorylation of specific tyrosine residues, leading to the activation of signal transduction. The key event in c-Met-mediated HGF signal transmembrane transduction is the phosphorylation of tyrosine residues. However, at present, it is unknown whether all the tyrosine residues are phosphorylated following ligand binding or only specific tyrosine residues. If the latter hypothesis is correct, factors affecting the phosphorylation of cMet at specific tyrosine residues require determination, as well as the effect of different ligands on the phosphorylation of specific residues. In addition, the correlation between the phosphorylation of tyrosine residues and the activation of intracellular signal transduction pathways, and the molecular mechanisms involved in the regulation of tumor metastasis require investigation. Clarification of these issues is likely to contribute to the understanding of the molecular mechanisms of transmembrane growth factor receptor-mediated signal transduction and tumor metastasis (9).

To study the molecular mechanisms of cMet-mediated cell signal transduction in the regulation of lymph node metastasis of liver cancer cells, the effect of HGF, FN and LN on the phosphorylation of c-Met at different tyrosine residues was compared in two cell lines. Our results showed that, following HGF treatment, the phosphorylation of cMet at Tyr 1313 and 1365 in highly metastatic Hca-F cells was higher, whereas the phosphorylation of cMet at Tyr 1349 was lower than that in low metastatic Hca-P cells. In addition, the activity of the PLC $\gamma / D A G / P K C$ signaling pathway was greater in Hca-F cells compared with Hca-P cells; however, the activity of the PI3K/AKT signaling pathway was lower.

$\mathrm{FN}$ is an important cell adhesion molecule involved in cell adhesion during tumor metastasis and has been reported to directly or indirectly affect cMet activation, thus regulating tumor metastasis (13). Results of the current study demonstrated that, following FN treatment, the phosphorylation of cMet at Tyr 1313 and the activity of the PLC $\gamma / D A G / P K C$ signaling pathway in highly metastatic Hca-F cells were higher than that in low metastatic Hca-P cells.

$\mathrm{LN}$ is also an important cell adhesion molecule involved in cell adhesion and the regulation of c-Met activation and phosphorylation of tyrosine residues (13). The results of this study indicated that, following LN treatment, the phosphorylation of cMet at Tyr 1365 and activity of the PLC $\gamma /$ DAG/PKC signaling pathway in Hca-F cells were higher than that in Hca-P cells.
In conclusion, under stimulation with various ligands, phosphorylated tyrosine residues of c-Met and the activated intracellular signal transduction pathways were found to vary in the same cell line. We hypothesized that the molecules involved in tumor metastasis were different at various stages and their expression required the regulation of different intracellular signal transduction pathways. Therefore, at different stages in the process of tumor cell metastasis, different ligands bind to cMet, leading to the phosphorylation of different tyrosine residues and activation of different intracellular signal transduction pathways, which accommodates the various processes involved in the regulation of tumor cell metastasis.

Under stimulation by the same ligand, the phosphorylated tyrosine residues of c-Met and the activation of intracellular signal transduction pathways were different between Hca-P and Hca-F cells. We hypothesized that these observations may be associated with differences in membrane structure and the microenvironment in which c-Met resides between the two cell lines. On the cell surface, growth factor receptors often combine with specific lipids (i.e., glycosphingolipids), membrane proteins and intracellular signaling molecules to form a specific membrane structure or microdomain, including sugar synapses (glycosynapse) or lipid rafts. Growth factor receptor-mediated signal transmembrane transduction is not only the result of ligand interaction with the receptor, but also the result of complex interactions of the receptor with other membrane components, including membrane lipids and proteins. On the cell surface, cMet is often enriched in specific membrane microdomains with four transmembrane proteins: CD82, CD9, integrins and tyrosine protein kinases $(14,15)$. In addition, specific molecules, including gangliosides, GM2/GM3, CD82 and CD9, inhibit the activation of c-Met $(16,17)$. Our previous studies also found that the expression of gangliosides in the two cell lines used in the present study was significantly different (18), which may explain why different tyrosine residues were phosphorylated following c-Met activation using the same ligand, which then activated different intracellular signal transduction pathways in the two cell lines.

After cMet is activated, different tyrosine residues are phosphorylated, which leads to the activation of the PI3K/AKT and PLC $\gamma / D A G / P K C$ signaling pathways to various extents in the two cells lines. These observations may result in the different lymph node metastatic potentials of the two cell lines.

In the current study, analysis of the relationship between the corresponding changes in the phosphorylation of $\mathrm{cMet}$ at different tyrosine residues and the activity of various signaling pathways in Hca-P and Hca-F cells suggested that the phosphorylation of cMet at Tyr 1313 and 1365 was associated with PLC $\gamma / \mathrm{DAG} / \mathrm{PKC}$ signaling pathway activity and the phosphorylation of cMet at Tyr 1349 may play a role in the activity of the PI3K/AKT signaling pathway. Furthermore, HGF, FN and LN were demonstrated to modulate cell motility and migration via the PLC $\gamma / D A G / P K C$ signaling pathway.

The mechanisms of cMet phosphorylation and the regulation of tumor metastasis appear to be complex and require further study for a more complete understanding of these processes. 


\section{Acknowledgements}

The current study was supported by grants from the National Program on Key Basic Research Project (973 program; no. 2012CB822103) and Liaoning Province Education Bureau (no. 2009T022).

\section{References}

1. Edakuni G, Sasatomi E, Satoh T, et al: Expression of the hepatocyte growth factor/c-Met pathway is increased at the cancer front in breast carcinoma. Pathol Int 51: 172-178, 2001.

2. Yamaguchi R, Yano H, Iemura A, et al: Expression of vascular endothelial growth factor in human hepatocellular carcinoma. Hepatology 28: 68-77, 1998.

3. Salgia R: Role of c-Met in cancer: emphasis on lung cancer. Semin Oncol 36 (2 Suppl 1): S52-S58, 2009.

4. Mitra AK: Ligand-independent activation of c-Met by fibronectin and $\alpha(5) \beta(1)$-integrin regulates ovarian cancer invasion and metastasis. Oncogene 30: 1566-1576, 2011.

5. Sourbier C: Met and the microenvironment: new insights for ovarian cancer metastasis. Cell Adh Migr 5: 209-210, 2011.

6. Kawakami Y, Kawakami K, Steelant WF, et al: Tetraspanin CD9 is a 'proteolipid,' and its interaction with alpha 3 integrin in microdomain is promoted by GM3 ganglioside, leading to inhibition of laminin-5-dependent cell motility. J Biol Chem 277: 34349-34358, 2002.

7. Sorokin AV, Mikhailov AM, Kachko AV, et al: Human recombinant laminin-binding protein: isolation purification and crystallization. Biochemistry (Mosc) 65: 546-553, 2000

8. Toledo MS, Suzuki E, Handa K and Hakomori S: Effect of ganglioside and tetraspanins in microdomains on interaction of integrins with fibroblast growth factor receptor. J Biol Chem 280 $16227-16234,2005$
9. Birchmeier C, Birchmeier W, Gherardi E and Vande Woude GF: Met, metastasis, motility and more. Nat Rev Mol Cell Biol 4: 915-925, 2003

10. Sun P, Wang XQ, Lopatka K, Bangash S and Paller AS: Ganglioside loss promotes survival primarily by activating integrin-linked kinase/Akt without phosphoinositide 3-OH kinase signaling. J Invest Dermatol 119: 107-117, 2002.

11. Yu H,Fukami K, Itoh T and Takenawa T: Phosphorylation of phospholipase Cgammal on tyrosine residue 783 by platelet-derived growth factor regulates reorganization of the cytoskeleton. Exp Cell Res 243: 113-122, 1998 .

12. Jeffers M, Rong S and Vande Woude GF: Hepatocyte growth factor/scatter factor-Met signaling in tumorigenicity and invasion/metastasis. J Mol Med (Berl) 74: 505-513, 1996.

13. Wang $\mathrm{J}$ and Geng $\mathrm{X}$ : HGF/cMet signaling pathway in hepatocellular carcinoma invasion and metastasis. J Clin Invest 18: 731-732, 2002.

14. Liu WM and Zhang XA: KAI1/CD82, a tumor metastasis suppressor. Cancer Lett 240: 183-194, 2006.

15. Miyake M, Koyama M, Seno M and Ikeyama S: Identification of the motility-related protein (MRP-1), recognized by monoclonal antibody M31-15, which inhibits cell motility. J Exp Med 174: 1347-1354, 1991

16. Todeschini AR, Dos Santos JN, Handa K and Hakomori SI: Ganglioside GM2-tetraspanin CD82 complex inhibits met and its cross-talk with integrins, providing a basis for control of cell motility through glycosynapse. J Biol Chem 282: 8123-8133, 2007.

17. Ono M, Handa K, Sonnino S, et al: GM3 ganglioside inhibits CD9-facilitated haptotatic cell motility: coexpression of GM3 and CD9 is essential in the downregulation of tumor cell motility and malignancy. Biochemistry 40: 6414-6421, 2001.

18. Huang X, Li Y, Zhang J, Xu Y, Tian Y and Ma K: Ganglioside GM3 inhibits hepatoma cell motility via down-regulating activity of EGFR and PI3K/AKT signaling pathway. J Cell Biochem 114: 1616-1624, 2013. 